\title{
Prehospital and Disaster Medicine: Roles and Responsibilities
}

\author{
Marvin L. Birnbaum, MD, PhD
}

\section{... To know what is impenetratable to us really exists manifesting itself as the highest wisdom and the most radiant beauty...}

Albert Einstein

...The mind is restless, turbulent, strong, and unyielding... As difficult to subdue as the wind...

Bhagavad-bita

Given the rapidly changing world with the ever-increasing frequency and severity of disasters, the progressive saturation of emergency medical care delivery systems, and the recognition of the vital role of public health in catastrophes, the demands and expectations placed upon disaster and emergency medical personnel are becoming greater than ever. Thus, it seems cogent to examine the roles and responsibilities of Prebospital and Disaster Medicine (PDM) in the current state of the world. The perceptions of this journal's responsibilities that follow are those of this editor who has had the honor and privilege of serving the readers for the last 14 years. Hopefully, this information will broaden your understanding of the skeleton of your journal.

\section{Provide Access to Information}

The provision of access to relevant information is the principal raison d'etre for any scientific publication. This may be confounded when the information provided serves a readership that crosses international borders and cultures. The information that affects our practice is multidimensional and often originates in meetings and congresses that most of the readership is unable to attend. In order to disseminate this information, PDM publishes papers and especially abstracts of scientific assemblies and congresses that otherwise would be inaccessible to many readers. Although the principal scientific language used for most scientific publications is English, for many readers, English is not their primary language. Therefore, PDM publishes many of the abstracts in languages other than English (thus far, in Spanish thanks to Daniel Rodriguez and Felipe CruzVega), but in the near future, additional languages will be added). The recognition that English is not the first language of many of the readers places certain constraints on how the English is structured in PDM. The style selected is aimed specifically at ease of translation by those whose primary language is not English.

Two other mechanisms have been implemented to enhance the utility of PDM in the distribution of relevant knowledge. The first has been the initiation of the Reference Clearing House ( $\mathrm{RCH})$. The $\mathrm{RCH}$ is available on the PDM web site (http://pdm.medicine.wisc.edu), and is a compilation of all of the references used in the articles published in PDM. When such references refer to abstracts published in Medline or other databases, direct links are provided to the abstracts. In addition, $\mathrm{MesH}$ headings are provided so that the entire Reference Clearing House is accessible through use of the PDM Search Engine.

Second, since many persons interested and dependent upon the information provided by PDM are not able to obtain the printed version of the journal, an on-line version of PDM is being provided. Volume 16, Number 4 (October-December 2001) and Volume 17, Number 1 (January-March 2002) have been provided with free access to all on the PDM web site. Hereafter, access to the on-line version of PDM will be password protected. Each subscriber (including members of the sponsoring organizations) will be given a User's Name and Password; the web-based version of each issue will be posted on the web site approximately onemonth before distribution of the printed version. If sufficient support can be obtained, User's Names and Passwords will be provided to persons who cannot otherwise access the information. Six months after publication and distribution of the printed issue, free access will be given to all who access the PDM web site. Mechanisms are being developed to allow subscriptions to only the web-based version of the journal at a reduced price to the subscriber.

\section{Advance Our Science}

Prehospital, emergency, and disaster medicine is a relatively young discipline with its science still in its infancy. PDM has the responsibility for the codification of this science worldwide. It must contribute to the development of the science by: 1) Providing the structure for the conduct and reporting of research; 2) Stimulating research in areas of need; 3) Facilitating the growth of young investigators; 4) Assuring what is published is valid, correct, and relevant to the development of the science; 5) Evolving a set of common definitions; and 6) Assisting in establishing standards and guidelines for the discipline.

Development of our science has been hampered by the lack of an accepted, standardized set of methodologies that can be applied to this discipline. For disaster medicine, the use of traditional medical research methodologies is difficult, 
if not impossible. Much has been published about the addition of research techniques that are standard for the study of the social sciences. This process will be furthered by the publication of an instructional series on the use of appropriate descriptive and qualitative techniques in medical research. This series will begin in the next issue of the journal. Further standards and guidelines will be published as part of the Health Disaster Management: Guidelines for Evaluation and Research in the Utstein Style that will be published as Supplements to this and the next volume of PDM.

The Guidelines also will provide the structure needed for the conduct and reporting of disaster medical and public health research to which the publications in PDM will adhere. In addition, the Guidelines will provide a comprehensive Glossary of Terms and the Conceptual Framework necessary to promote the interdisciplinary understanding of catastrophic events and the disasters that result.

Members of the Editorial Board of PDM and the other peer-reviewers that assist the Board have the ongoing responsibility for assuring the validity and correctness of what gets published. The relevance of what is published will be developed further by the resumption of the Editorial Comments following each article published. This feature was included in earlier editions of PDM and is being resumed at the request of the readership. Dr. Sam Stratton, who has been a member of the Editorial Board for seven years, has accepted the responsibility for resumption of the Editorial Comments. These comments will be particularly relevant to our ability to interpret the data/information published in light of our associated disciplines. We must integrate our science with the knowledge and expertise of the related disciplines including management, engineering, media, education, logistics, security, politics, social services, international law, etc.

\section{Broaden Our Knowledge Base}

The disciplines supported by PDM necessarily are broadso broad that it is not possible for us to maintain our knowledge only through the digestion of that with which we are most familiar. In order to broaden our knowledge base and our appreciation for our fellow professionals, it is helpful for us to have access to some of the knowledge-base and perceptions of associated professionals. PDM attempts to help fill such gaps through the publication of theme issues that summarize the state-of-the-art in those areas that may not fall within our own area of expertise. Experts with a comprehensive knowledge base in a particular area are selected by Theme Editors whose expertise allows them to select contributors who are the most expert known in the field. Thus far, two theme issues have been published: 1)
Disasters and Public Health (Volume 15 (4), and 2) Complex Emergencies (Volume 16 (4)). Two additional special theme issues will be published in the near future: 1 ) Terrorism (Jeff Arnold, Editor); and 2) Psychosocial Aspects of Disasters (Gloria Leon, Editor).

Lastly, a special area of interest to us all and for which most of us have very little expertise and knowledge is international health law (IHL). A special Section of PDM will be devoted to IHL. This Section will be organized and edited by Mike Hoffman, currently Counsel for the American Red Cross.

\section{Foster Relationships with Industry Partners}

Manufacturers of the supplies and equipment upon which we are dependent must be viewed as our partners in practice. This industry is important not only for its contributions to the support of the Journal, but for supplying the equipment and supplies we need to practice. Thus, it is incumbent upon us to support the industry as well. Our support must come in the form of providing consultation for the development of new products necessary to improve practice and assisting in the testing and distribution of such products. This has been the prevailing philosophy of this Journal since the beginning of my tenure as editor and such efforts will be intensified in the future.

The aforementioned are some of the standards by which PDM is to be judged. The responsibilities of PDM are great. The Editorial Board assures that these responsibilities are met without bias and in accordance with the policies it establishes. The Editorial Board is broadly based and is responsible for what your Journal provides. It crosses disciplines and cultures. Its charge is to provide you with the information essential to the enhancement of your practice. When reading PDM, it is important to keep in mind all of the responsibilities outlined above. Some of what you peruse will be more relevant to your practice than others. But, the scope necessarily is broad and must reach across disciplines, borders, and cultures. As always, your comments and suggestions are welcome.

The wise man looks into space, And does not regard the small as too little,

Nor the great as too big;

For be knows that there is no limit to dimensions.

With all beings and all things, we shall be relatives.

Lao-tse

American Sioux Indian

[all quotes taken from The Family of Man. Steichen E (ed), New York: Maco Magazine Corp. 1955.] 